\title{
A three-way perspective of stoichiometric changes on host-parasite interactions
}

\author{
Sanni L. Aalto ${ }^{1}$, Ellen Decaestecker ${ }^{2}$, and Katja Pulkkinen ${ }^{1}$ \\ ${ }^{1}$ Department of Biological and Environmental Science, University of Jyväskylä, PO Box 35, 40014 Jyväskylä, Finland \\ ${ }^{2}$ Laboratory of Aquatic Biology, Department of Biology, Katholieke Universiteit Leuven Kulak, Etienne Sabbelaan 53, 8500 Kortrijk, \\ Belgium
}

\begin{abstract}
Changes in environmental nutrients play a crucial role in driving disease dynamics, but global patterns in nutrient-driven changes in disease are difficult to predict. In this paper we use ecological stoichiometry as a framework to review host-parasite interactions under changing nutrient ratios, focusing on three pathways: (i) altered host resistance and parasite virulence through host stoichiometry (ii) changed encounter or contact rates at population level, and (iii) changed host community structure. We predict that the outcome of nutrient changes on host-parasite interactions depends on which pathways are modified, and suggest that the outcome of infection could depend on the overlap in stoichiometric requirements of the host and the parasite. We hypothesize that environmental nutrient enrichment alters infectivity dynamics leading to fluctuating selection dynamics in host-parasite coevolution.
\end{abstract}

\section{Anthropogenic nutrient enrichment alters disease dynamics: how and why?}

Anthropogenic disturbances in global biogeochemical cycles of nitrogen $(\mathrm{N})$ and phosphorus $(\mathrm{P})$ have not only led to increased nutrient loading in the environment but also to changed carbon $(\mathrm{C})$ to nutrient ratios $(\mathrm{C}: \mathrm{N}: \mathrm{P})$ available to organisms. Based on the assumptions of ecological stoichiometry, C:N:P of resources are reflected in the stoichiometric content of the organisms consuming those resources (Box 1). For example, spatial imbalances in atmospheric loading of $\mathrm{N}$ cause spatial variation in $\mathrm{N}: \mathrm{P}$ ratios in organisms, with a shift towards higher $\mathrm{N}: \mathrm{P}$ in organisms in areas with high loading of $\mathrm{N}[1,2]$. Owing to fundamental differences in allocation of these three key elements $(\mathrm{C}, \mathrm{N}, \mathrm{P})$ to major biomolecules (e.g., nucleic acids, amino acids), or to chemical structures between organisms [3] and in their life-history strategies, changes in $\mathrm{C}: \mathrm{N}: \mathrm{P}$ ratios will either favor or impinge upon the function and fitness of organisms. The elemental ratio $(\mathrm{C}: \mathrm{N}: \mathrm{P})$ in a resource thus defines resource quality for a

\footnotetext{
Corresponding author: Aalto, S.L. (sanni.lh.aalto@jyu.fi).

Keywords: ecological stoichiometry; host-parasite coevolution; infection dynamics; nutrient ratios; resource quality.

$1471-4922 /$

(C) 2015 Elsevier Ltd. All rights reserved. http://dx.doi.org/10.1016/j.pt.2015.04.005
}

consumer. For example, decreases in environmental C:P ratios (i.e., increase in $\mathrm{P}$ ) are likely to favor fast growing organisms that allocate $\mathrm{P}$ strongly to $\mathrm{P}$-rich ribosomal RNA (rRNA), which is needed for protein synthesis and growth $[4,5]$. By changing population dynamics, increased nutrient loading has the potential to modify species interactions at different trophic levels and in different ecosystem functions $[2,6,7]$.

In recent years, increases in parasitic and infectious diseases have been connected to increased nutrient loading in the environment [8-10]. These studies have mainly focused on resource quantity-driven changes in disease dynamics, examining increased host production under conditions of nutrient enrichment. However, parasites can also be considered as consumers, and thus changes in their resource quality - in other words, in the stoichiometric content of hosts - could alter their fitness and function in similar ways as resource quality-driven changes in producer stoichiometric content affect herbivores (Box 1). Indeed, to predict how and why hosts and parasites respond at the individual, population, and ecosystem levels to changed ratios of elemental nutrients, the framework provided by ecological stoichiometry can be utilized (Box 1). Based on the assumptions of ecological stoichiometry, anthropogenic increases in $\mathrm{N}$ and $\mathrm{P}$ concentrations in the environment would not only support the growth of individual hosts and host populations but would also increase the nutrient content of hosts, thus increasing resource quality and availability to parasites. By contrast, increased nutrient levels could also facilitate host resistance and hence suppress parasite growth and transmission.

We review here what is known about the effects of environmental nutrient changes on host-parasite interactions in a stoichiometric framework. We focus on endoparasites, which exploit host tissues or storage biomolecules metabolized from the food (described e.g., in [11]). We propose a three-pathway perspective (Figure 1). Pathway 1 focuses on the interactions between individual hosts and parasites modified by nutrient levels. Pathway 2 describes how environmental nutrient levels change parasite transmission efficiency via altering host population density. With respect to pathways 1 and 2, we review the effects that changes in environmental nutrient ratios are likely to 


\section{Box 1. Ecological stoichiometry}

Sterner and Elser [100] define ecological stoichiometry as: 'the balance of multiple chemical substances in ecological interactions and processes, or the study of this balance. Also sometimes refers to the balance of energy and matter'.

The assumptions that ecological stoichiometry makes are based on the law of conservation of matter, which states that the 'amount of matter in a closed system is conserved' and that 'in an ordinary chemical reaction, the total mass of the products must equal the total mass of the reactants'. From the perspective of ecological stoichiometry, this means that all elements in the resources that an organism consumes must be converted either into biomass of that organism or into waste (Figure I) [100].

All organisms are built from three key elements: carbon (C), nitrogen $(\mathrm{N})$, and phosphorus $(\mathrm{P}) . \mathrm{C}$ is the main component of biomolecules involved in energy metabolism (e.g., lipids and carbohydrates), while $\mathrm{N}$ and $\mathrm{P}$ are constituents of biomolecules (e.g., nucleic acids, amino acids) and of functional and structural compounds [100]. In ecosystems, $\mathrm{N}$ and $\mathrm{P}$ are the usually the two elements that limit biomass production - in other words, are 'nutrients' - whereas $C$ is generally found in excess, meaning that the environmental C: $\mathrm{N}$ and C:P ratios are high [101]. Most studies on ecological stoichiometry have focused on $P$ because it is necessary for building essential compounds for life (DNA, RNA, and ATP) and is more commonly found to limit growth of organisms than $\mathrm{N}$ (as reviewed in [102]).

Each organism has an unique elemental content, in other words the ratio between carbon and nutrients $(\mathrm{C}: \mathrm{N}: \mathrm{P})$, reflecting the extent to which it allocates elements to major biomolecules and to chemical structures [3]. This again is related to the life-history strategy of the organism. Indeed, one of the central concepts in ecological stoichiometry, the growth rate hypothesis (GRH), suggests that fast-growing organisms have low $\mathrm{C}: \mathrm{P}$ and $\mathrm{N}: \mathrm{P}$ ratios (high $\mathrm{P}$ content) because they allocate $\mathrm{P}$ strongly to $\mathrm{P}$-rich ribosomal RNA (rRNA) which is needed for protein synthesis and growth [4,5]. GRH is most applicable to small heterotrophic P-limited organisms [102], and does not apply under special conditions: for example, under co-limitation of essential nutrients [103].

The connection between life-history traits and stoichiometric composition of an organism means that acquisition of $\mathrm{C}$ and elemental nutrients in imbalanced ratios impinges on the function

have on host-parasite coevolution. Pathway 3 then describes how nutrient-driven changes in the host community can modify host-parasite interactions.

\section{Pathway 1. Nutrient-driven within-host infection dynamics}

In pathway 1 we focus on the interactions at the level of a host individual (Figure 1). At the onset of the infection process, parasite infectivity will largely depend on factors other than host nutrients, such as genetic compatibility between the parasite and the host [12]. However, after successful onset and establishment of infection, the parasite is able to retrieve host resources and even to manipulate host resource allocation to acquire energy and nutrients. For example, host 'castrating' parasites make the host divert resources from reproduction to growth, which the parasites in turn exploit for their propagule production $[13,14]$. Some microbes have been found to trigger biosynthesis and degradation pathways of the hosts to acquire essential biochemical compounds $[15,16]$.

Following the assumptions of ecological stoichiometry, higher environmental nutrient levels can be expected to cascade via the food web from primary producers into increased resource quality for consumer-hosts. However, whether these higher-quality hosts (higher resource and fitness of the organism. Each organism has a defined threshold elemental ratio (TER) after which it transitions from energy limitation to elemental limitation [102]. Some organisms need to maintain constant chemical compositions under varying resource quality (resource $\mathrm{C}: \mathrm{N}: \mathrm{P}$ ) - in other words, they are more stoichiometrically homeostatic than others. Autotrophs are generally considered to be less homeostatic than consumers and suffer less from imbalanced resource quality because they can incorporate excess $C$ into structures and storage matter. Consumers are considered to be more stoichiometrically homeostatic. They allocate resources strongly towards nucleic acids and to other $\mathrm{N}$ - and P-rich compounds needed for growth and reproduction, and consequently need to maintain high nutrient content and require high quantities of nutrients (low C:N:P) [100]. When consuming resources with imbalanced $C: N: P$, they suffer from reduced function and fitness and need to actively remove excess C [102]. Previous studies in ecological stoichiometry have focused on producer-herbivore interactions because there is significant stoichiometric mismatch between plant and animal tissue. However, poor resource quality (high $\mathrm{C}: \mathrm{N}: \mathrm{P}$ ) may cascade up to the food chain to constrain secondary and tertiary consumers [104], and possibly parasites [28]

Water fleas from the genus Daphnia have been the main study organisms in ecological stoichiometry because they are common herbivores in aquatic ecosystems and have high $\mathrm{P}$ requirements, meaning that they commonly suffer from P-limitation [101].

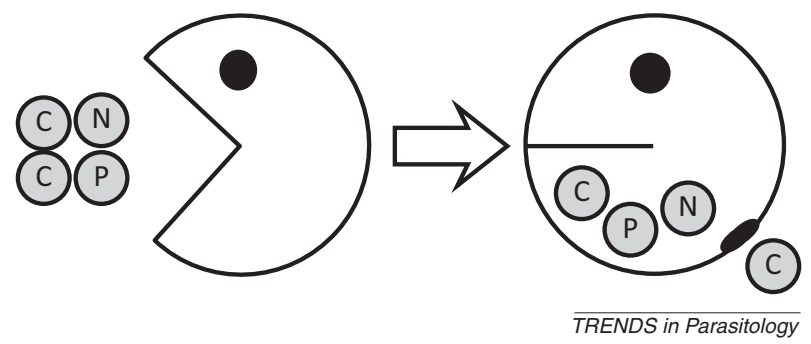

Figure I. Law of conservation of matter in consumer-resource interactions. All the elements gained are either incorporated into new biomass of the consumer or are secreted as waste.

quality for parasites) will result in increased parasite fitness is determined by resource allocation between host resistance and parasite growth. If the host is able to invest elemental resources into resistance before parasite exploitation, higher resource quality (low $\mathrm{C}: \mathrm{N}$ or $\mathrm{C}: \mathrm{P}$ ) could lead to decreased parasite load, even though such resistance mechanisms may be costly to the host [17]. In accordance, a nutrient-supplemented high-quality diet has been documented to lead to increased immune activity and/or reduced parasite multiplication rate (e.g., [18-23]). However, if the parasite is able to steal the nutritional elements before the host can allocate them to immune defense, higher host quality (high amount of $\mathrm{P}$ and/or N) can lead to increased parasite growth rate and production of infective propagules within individual hosts (Figure 2). Higher nutrient ( $\mathrm{N}$ and/or $\mathrm{P}$ ) availability has been found to promote the replication of various parasites, including fungal foliar pathogens in plants [24], bacteria and fungi in corals $[25,26]$, trematodes in snails [27], and bacteria and fungi in water fleas $[28,29]$. In addition, host resource-driven increases in parasite replication rate have been found to enhance the negative effect of parasites on host fitness (i.e., parasite virulence) $[20,30]$. Parasite fitness may also depend on host tolerance, in other words the ability of the host to maintain normal physiological functions when 


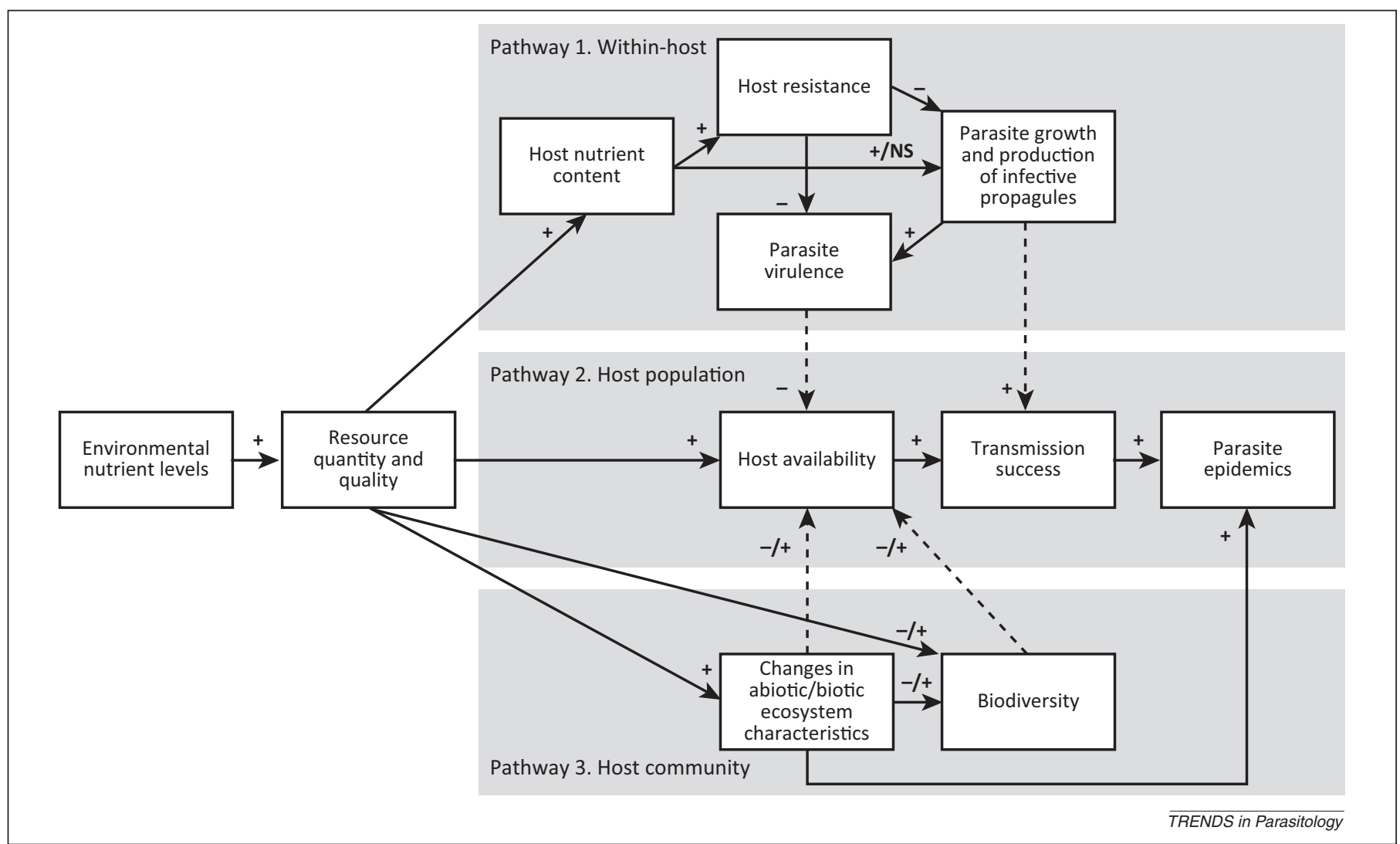

Figure 1. Schematic representation of the three suggested pathways describing the mechanisms by which environmental nutrient levels may alter parasite epidemics in nature. In pathway 1, environmental nutrient levels drive changes in parasite infection patterns through altering host resistance and parasite virulence; in pathway 2 , through host availability; and in pathway 3, through shaping host communities (+, positive effect; -, negative effect; NS, non-significant effect). Broken arrows indicate connections between different pathways. Unbroken arrows indicate within-pathway connections.

infected. Increase in resource quantity has been demonstrated to increase host tolerance [30], and high resource quality could be expected to have a similar effect if hosts are able to allocate increased nutrients to increased tolerance. From a stoichiometric perspective, however, hosts may have innate differences in their ability to tolerate parasite-driven nutrient depletion owing to differences

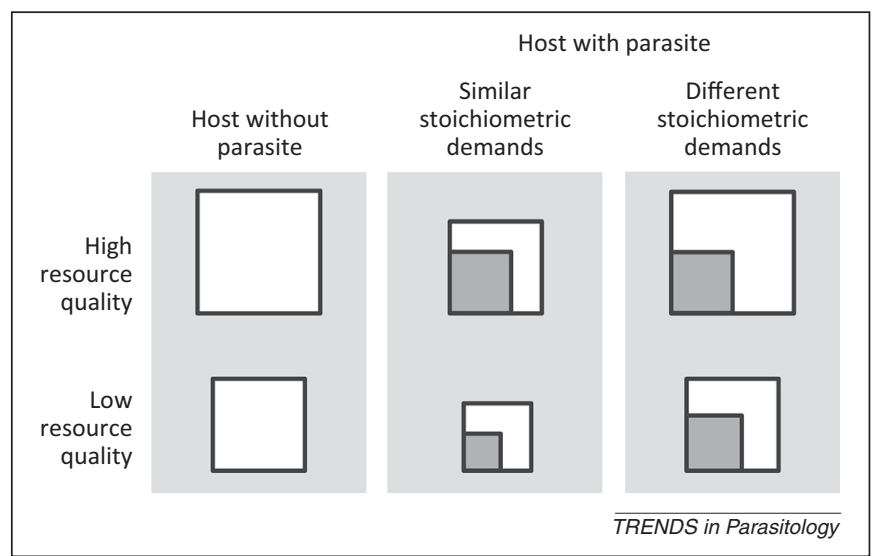

Figure 2. A mechanistic explanation of the effect of host resource quality on within-host parasite growth and virulence. The white square represents the host and the grey square the parasite within the host. Box size reflects the growth response of the host or the parasite. In the absence of the parasite, low resource quality reduces host fitness. Under similar stoichiometric demands of parasite and host, the parasite shows high growth rate and virulence under high resource quality conditions, and low growth rate and virulence under poor resource quality conditions. By contrast, if parasite and host have different stoichiometric demands, parasite could show low virulence regardless of resource quality. in the level of homeostasis (Box 1). Less-homeostatic hosts might suffer less from elemental exploitation of parasites than strictly homeostatic hosts. Currently, however, there are no studies concerning resource quality-driven changes in host tolerance.

Differences in parasite life-history strategies can be expected to lead to differences in the stoichiometric demands of the parasites and hence to different host exploitation strategies (Figure 2) [31]. For example, fastgrowing parasites should have higher requirements for $\mathrm{P}$ (growth rate hypothesis, Box 1), and they would therefore preferentially exploit either ingested $\mathrm{P}$ or P-rich tissues of their hosts. Whether the hosts then suffer from decreasing $\mathrm{P}$ availability or not depends on their stoichiometric demands. We suggest that, in addition to resource allocation between the host and the parasite, the outcome of infection can be expected to depend also on the overlap in the stoichiometric requirements between the host and the parasite - in other words, the strength of their resource competition (Figure 2) [32,33]. Under high nutrient input, parasites with high nutrient demands will show a high replication rate and high virulence, while upon nutrientdeficiency of the host their replication rate will be depressed. This expectation is based on the model of evolution of virulence stating that the rates at which parasites extract resources from their hosts equates to their virulence [34]. By contrast, if stoichiometric demands of host and parasite differ - for example, if the parasite has lower or different requirements for nutrients than the host - then 
the parasite could show low virulence (or the host could show high tolerance) independently of parasite replication rate (Figure 2). For example, the parasites of Daphnia water fleas that depend on host nutrient content $(\mathrm{N}$ and/or P) show high virulence $[28,29]$, while a benign microsporidian parasite Glugoides intestinalis is not fostered by host $\mathrm{P}$ enrichment, but possibly by increased host carbon intake (food quantity) [33,35].

In multiple infections, the combined negative effect of the parasites will depend on whether the parasites require nutrients that are essential for the host. If multiple infections involve both a parasite that depends on the essential and a parasite that depends on the non-essential nutrients for the host, the outcome of the infection might not differ from single infections with a parasite dependent on the essential nutrients [36]. Furthermore, the type of interaction between the parasites will be important: antagonistic interactions (e.g., competition for nutrients) between parasites can decrease virulence [37], while a reciprocal facilitation could lead to increased virulence levels [38]. This has not been studied directly but, in coinfection of plant Avena sativa with two virus species, low $\mathrm{N}$ supply to host plant led to one virus species, barley yellow dwarf virus species PAV (BYDV-PAV), outcompeting the other (cereal yellow dwarf virus species RPV), and this was explained by higher nutrient competition ability of BYDV-PAV supporting its higher replication rate [39,40]. Latent infections can benefit the host, preventing other infections from establishing or becoming more severe [41]. This can be food quality-dependent: the water flea Daphnia magna populations were protected against a virulent bacterial parasite by the less-virulent microsporidian parasite under low food quality conditions [42].

\section{Pathway 2. Nutrient-driven parasite transmission patterns through changes in host population-level responses}

At the population level, we suggest that changes in environmental nutrient levels are likely to alter transmission patterns mainly through changes in contact rates between infected and uninfected individuals, or in encounter rates between hosts and parasite propagules. First, high resource quality (low $\mathrm{C}: \mathrm{N}: \mathrm{P}$ ) may increase transmission by promoting the growth and reproduction of individual hosts, therefore increasing host density. This production-mediated effect on infection dynamics has been well established in nature and in several experimental host-parasite systems. For example, McKenzie and Townsend [43] presented several examples where increased nutrients ( $\mathrm{N}$ and/or $\mathrm{P}$ ) promoted parasite epidemics via increasing intermediate host or vector density. Johnson et al. [27] noticed that higher $\mathrm{N}$ and $\mathrm{P}$ input promoted trematode parasite Ribeiroia ondatrae infection in amphibians via enhanced algal production and increased density of the intermediate host, the snail Planorbella trivolvis. Colwell [44] explained the positive parallel patterns between environmental nutrient levels and cholera outbreaks in susceptible human populations via an increase in copepod abundance through nutrient loading. Copepods act as an attachment base for the cholera bacteria Vibrio cholerae. However, high resource quality (low $\mathrm{C}: \mathrm{N}: \mathrm{P}$ ) may increase transmission efficiency and parasite prevalence in host population also through increasing host stoichiometric quality, which then increases parasite growth (production of infective propagules as indicated in pathway 1) and host-parasite encounter rates $[27,45]$. In addition, increased parasite growth might lead to increased doses of parasite propagules being encountered by individual hosts [46], and this might increase the probability of infection but also curtail parasite production in that particular host [47], with contrasting effects on parasite epidemics.

In addition to host and parasite densities, encounter rates between hosts and parasites may be modified by indirect trait-mediated effects (TMIEs) such as host aggregation [48] and the feeding efficiency of individual hosts [49]. TMIEs have been associated with resource quality [50] and have been investigated substantially in tri-trophic plant-herbivorous insect-parasite systems where plant defense chemistry and nutrient content alter parasite transmission $[51,52]$. Furthermore, changes in host resource quality can change the quantity or quality of host excreta that some parasites use for host localization [53], again affecting infection probability determined by hostparasite encounter rates. Different stoichiometric requirements between the host and parasites might also change the encounter rates in ways not anticipated from hostparasite interactions at the individual host level. In a recent experiment [35], nutrient limitation decreased host density but did not alter parasite within-host production, leading to higher per capita exposure rates and increased parasite intensity in host populations under nutrient limitation.

When enhancing parasite growth, environmental nutrient enrichment may also increase parasite virulence, which will decrease host population production and have a negative impact on infection dynamics via decreased encounter rates. However, high resource quality (low $\mathrm{C}: \mathrm{N}: \mathrm{P})$ may also enhance host fecundity compensation for fitness losses, and a nutrient-induced increase in virulence therefore might not immediately manifest itself as negative effects at the host population level [54]. For example, in a recent study one particular water flea species, Daphnia magna, was shown to be more susceptible to infections at high nutrient levels, but this was compensated by better growth conditions [55]. Nutrient-induced increases in parasite intensity might also be translated into higher mortality of the oldest/largest host individuals, releasing resources for compensatory population growth, and paradoxically increasing host density.

\section{Nutrient-driven changes in host-parasite coevolution}

Changes in environmental resource quality $(\mathrm{C}: \mathrm{N}: \mathrm{P})$ not only modify the epidemiology of host-parasite interactions but are also suggested to change the strength and direction of selection in host-parasite interactions [56,57]. If environmental nutrient enrichment is translated into higher population sizes, then increased host-parasite interactions, in terms of within-host competition and parasite transmission (pathways 1 and 2), may intensify the coevolutionary arms race between hosts and parasites. Evolutionary host population responses with respect to resistance towards parasites are assumed to be strongest 
in highly-productive systems [58-60]. Larger host and parasite populations broaden the genetic variation available for selection and increase encounter rates between hosts and parasites [61]. Higher encounter and transmission rates are associated with more-virulent parasite strains [34], which pose stronger selection pressures for host defenses, further increasing the selection pressure on parasites for counter-defense [62]. High environmental nutrient levels are found to favor P-rich and rapidly-growing species (growth rate hypothesis, GRH; Box 1) [6,63] which would, following pathway 1 , then offer high-quality resources for parasites, and this could again favor rapidlygrowing parasites that have high $\mathrm{P}$ demands and consequently express high virulence (as described above). Fast growth rates can be expected to intensify coevolutionary cycles via accelerating population turnover and increasing the number of mutations. Thus, environmental nutrient enrichment is expected to direct host evolution towards higher estance, and parasite evolution towards higher virulence 59,64], intensifying the coevolutionary arms race and reducing the period of coevolutionary frequency-dependent oscillations [65]. Nutrient availability may also affect host tolerance [23]. Because tolerance does not diminish parasite within-host growth, it is thought to impose less selection pressure on parasite virulence than resistance does [66]. However, if increased tolerance leads to increased parasite loads, prevalence, and transmission $[67,68]$, it may promote evolution towards elevated virulence [68].

Changes in resource quality have been demonstrated to drive shifts in genotype frequencies [69-72]. For example, P-rich resources favored water flea Daphnia pulicaria clones with higher $\mathrm{P}$ demands (higher $\mathrm{P}$ homeostasis) whereas, under P-depleted conditions, clones having lower requirements for $\mathrm{P}$ could outcompete $\mathrm{P}$-rich ones [70]. Based on this, we suggest that nutrient enrichment could favor and drive selection for particular host-parasite genetic combinations. In this scenario, environmental nutrient enrichment could increase the frequency of nutrient-rich hosts, and this consequently would increase the frequency of nutrient-rich parasite genotypes - and these would, as suggested in pathway 1 , be virulent (Figure 3). However, intense selection imposed by parasites might eliminate nutrient-rich hosts quickly, providing an opportunity for less nutrient-rich host genotypes with intermediate growth rates to increase in frequency (Figure 3). These nutrient-intermediate host genotypes could then favor less-virulent parasite genotypes with low nutrient demands, or the host might also be less affected, even if exploited by virulent parasite genotypes with high nutrient demands, because those would not be able to grow as aggressively as in nutrient-rich host genotypes. Nevertheless, if nutrient-rich host genotypes were able to recover, they could rapidly increase and outcompete nutrient-intermediate host genotypes under nutrient-enriched conditions [70]. We expect these recovery periods to shorten with increasing nutrient levels, implying that environmental nutrient enrichment might increase the frequency of genotype fluctuations - in other words, intensify frequency-dependent selection (Figure 3).

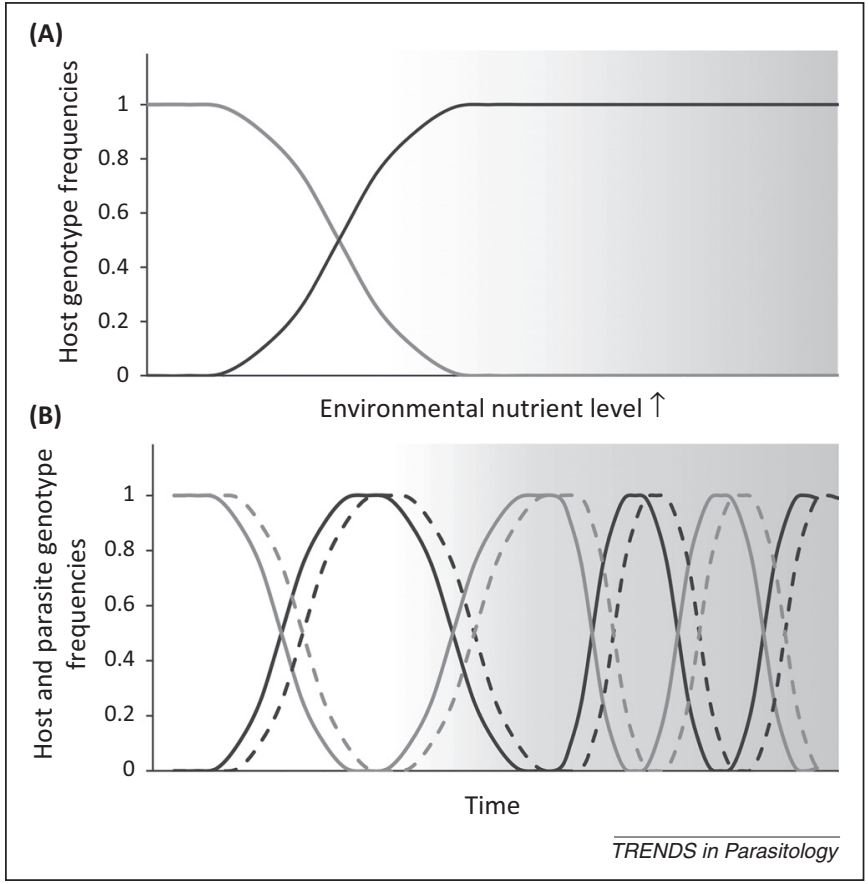

Figure 3. Effects of environmental nutrient enrichment on (A) host genotypes and (B) their coevolutionary oscillations with parasite genotypes. Black unbroken line, nutrient-rich host genotype; grey unbroken line, nutrient-intermediate host genotype; black broken line, nutrient rich parasite genotype; grey unbroken line, nutrient-intermediate parasite genotype.

\section{Pathway 3. Nutrient-driven infection dynamics at the host community level}

Next to effects on the host density, nutrient-driven changes in parasite infection dynamics can be even more complex and are structured via changes in trophic patterns in the host community composition. Environmental resource quality (C:N:P) can alter disease patterns via changes in species diversity and host availability because particular nutrient levels favor only some species as a function of to their stoichiometric demands and level of homeostasis (Box 1). For instance, $P$ enrichment has been found to promote malaria transmission via altering plant community structure to favor the more-efficient mosquito vector of malaria, Anopheles vestipennis, over the less-efficient $A$. albimanus [73]. In addition, Turner et al. [74] showed recently that nutrients released from carcasses infected by Bacillus anthracis improved vegetation growth and attracted herbivores to contaminated spots, thereby increasing the potential for transmission.

The pattern between resource quantity and taxa richness is commonly found to be hump-shaped [75], indicating that an intermediate increase in nutrient input could be expected to increase biodiversity. Increased biodiversity may modify infection dynamics via dilution effects, whereby increased numbers of non-competent hosts act as sinks for the disease and thus decrease infection prevalence [7680]. The effect of increased biodiversity on disease prevalence might, however, depend on the scale of examination $[81,82]$. In contrast to positive biodiversity response at intermediate nutrient levels, high resource nutrient content has also been found to reduce biodiversity (e.g., [83]), for example by favoring rapidly-growing P-rich species that are able to outcompete others $[6,63]$. This could lead to 
increased transmission rate and infection prevalence if the favored P-rich species are competent hosts.

Environmental resource quality $(\mathrm{C}: \mathrm{N}: \mathrm{P})$ may also increase or decrease the survival of transmission propagules independently of the host population or community. Brown et al. [84] suggested that adaptation to the outside-host environment may pre-adapt an opportunistic pathogen for virulent growth within a susceptible host. This was observed in a fish microbial community, where originally benign microbes were transformed into virulent ones in a nutrient-rich environment [85]. Penczykowski et al. [86] illustrated that an increase in nutrient levels supported the onset and size of fungal epidemics in lake zooplankton, and this was mediated by both abiotic and biotic interactions. Higher nutrient input led to a shallower light-penetration, which facilitated disease-spreading predators and UV-sensitive parasite production. Dallas and Drake [29] observed that high environmental nitrate levels increased the mortality of fungal spores and reduced the transmission of a fungal pathogen in a Daphnia population.

Further, it is important to note that nutrient-mediated changes in host-parasite interactions and in their coevolution may shape trophic dynamics and ecosystem level processes. For example, high resource quality may lead to higher parasite-mediated apparent competition if one high-quality host species supports higher parasite production, which then has a greater detrimental effect on other host species (e.g., [87]). In addition, high resource quality may reduce interspecific resource competition between host and non-host species if there is a resource qualitydriven increase in parasite virulence (see pathway 1). Furthermore, nutrient-induced increase in within-host parasite growth might decrease the stability of host populations [88], and consequently lead to sudden collapse of parasite populations [89], especially in presence of selective predators [90], and this might again alter trophic dynamics. In addition, nutrient-driven changes in hostparasite dynamics may alter nutrient cycling. Because parasites can alter host stoichiometry [91-93] and diet choice (e.g., $[18,22,94,95])$, they are likely to change the nutritional value of hosts for higher consumers [91] or the flux of elemental nutrients through the host [96]. Higher environmental nutrient levels could accentuate parasitedriven changes in nutrient cycling, if they are expected to lead to increased parasite epidemics, or to drive selection towards host-parasite genotypic combinations with high nutrient demands.

\section{Concluding remarks and future perspectives}

Each of the perspectives presented here highlights that interactions between environmental nutrient ratios and host-parasite dynamics are diverse and depend on several factors at the within-host (pathway 1), host population (pathway 2), host community, and ecosystem levels (pathway 3 ). We argue that even though host resource quality can shape the onset and progression of infection in individual hosts (pathway 1 ), the ultimate outcome depends on the similarity of stoichiometric demands between the host and the parasite. Currently we have only limited understanding of the mechanisms by which changes in environmental nutrient levels can modify infection dynamics at the within-host-level, and knowledge is even scarcer at the population and community levels. Outstanding questions for future research are proposed in Box 2.

Determining parasite species- or genotype-specific stoichiometric requirements with, for example, radioisotope technique [97,98], and comparing these against the lifehistory traits of the parasites, could help to resolve whether the assumptions of ecological stoichiometry can be applied to host-parasite interactions. In particular, experimental evidence on the connections between host resource quality, parasite virulence, and host resistance or tolerance could provide interesting new perspectives on hostparasite studies which have primarily focused on the parasite response to nutrient-increased host production. For this, traditional life-table experiments with host-parasite combinations having different stoichiometric demands could be applied, but one could also utilize next-generation methods - for example to follow parasite-induced changes in host gene expression as a function of nutrient use, or differential parasite gene expression under different host resource regimes - using functional genomics [99]. Knowledge of the connections between stoichiometric demands and infection traits would provide interesting opportunities for experimental studies concerning mechanisms at the host population and community levels, and for examining how environmental nutrients alter host-parasite co-evolution dynamics - for example, whether nutrient enrichment intensifies arms-race dynamics and negative frequency-dependent selection. The final goal would be to find an efficient genetic method which could be used for rapid screening of infection patterns in natural host-parasite communities under different nutrient regimes.

Detailed knowledge on disease under different nutrient regimes could also provide a tool for disease management given that there are likely to be positive connections between environmental nutrient levels and parasite prevalence, decreases in anthropogenic nutrient input could suppress disease and parasite infections at the ecosystem level. The role of nutrients in controlling disease might be especially important in aquatic ecosystems, where

\section{Box 2. Outstanding questions}

- Are the stoichiometric demands of parasites linked to life-history traits, and are they parasite species- or genotype-specific?

- Does the growth rate hypothesis (GRH) apply to parasites?

- Does GRH apply to parasitized hosts?

- What types of connections are there between stoichiometric demands, parasite virulence, host tolerance, and host resistance?

- Is there innate stoichiometric tolerance? - in other words, does the degree of homeostasis define how tolerant the host is to parasite nutrient exploitation?

- Which factor - nutrient-driven increase in host production or in host quality - has the major role in controlling infection dynamics at the host population level?

- What is the role of nutrients other than $\mathrm{N}$ and $\mathrm{P}$ (e.g., $\mathrm{K}$ [105]) in regulating disease dynamics?

- How do changes in environmental nutrient quality alter hostparasite coevolution?

- Does nutrient enrichment decrease or increase biodiversity, and how does it affect infection dynamics?

- What tools can be used to examine nutrient-driven infection dynamics in nature? 


\section{nutrients accumulate through deposition and run-off from} terrestrial catchment.

\section{Acknowledgments}

Authors thank T. Hiltunen and L. Mikonranta for constructive comments. E.D.C. thanks the members of Aquatic Biology, the KU Leuven Kulak group for stimulating discussions on this topic. Funding was provided by the Emil Aaltonen Foundation, Life+ N-SINK project FI/ENV/597, and the Academy of Finland (260797) to Marja Tiirola (S.A.), by Academy of Finland project funding (260704) to Jouni Taskinen (K.P.) and by research grants START1/08/019, Belspo IAP project SPEEDY, P7/4, and Center of Excellence SEEDS PF/2010/007 from the KU Leuven Research Fund (E.D.C.)

\section{References}

1 Elser, J.J. et al. (2009) Shifts in lake N:P stoichiometry and nutrient limitation driven by atmospheric nitrogen deposition. Science 326, 835-837

2 Elser, J.J. et al. (2010) Atmospheric nitrogen deposition is associated with elevated phosphorus limitation of lake zooplankton. Ecol. Lett. $13,1256-1261$

3 Loladze, I. and Elser, J.J. (2011) The origins of the Redfield nitrogento-phosphorus ratio are in a homoeostatic protein-to-rRNA ratio. Ecol. Lett. 14, 244-250

4 Elser, J.J. et al. (2000) Biological stoichiometry from genes to ecosystems. Ecol. Lett. 3, 540-550

5 Elser, J.J. et al. (2003) Growth rate-stoichiometry couplings in diverse biota. Ecol. Lett. 6, 936-943

6 Singer, G.A. and Battin, T.J. (2007) Anthropogenic subsidies alter stream consumer-resource stoichiometry, biodiversity and food chains. Ecol. Appl. 17, 376-389

7 Bobbink, R. et al. (2010) Global assessment of nitrogen deposition effects on terrestrial plant diversity: a synthesis. Ecol. Appl. 20, 30-59

8 Johnson, P.T.J. et al. (2010) Linking environmental nutrient enrichment and disease emergence in humans and wildlife. Ecol. Appl. 20, 16-29

9 Smith, V.H. and Schindler, D.W. (2009) Eutrophication science: where do we go from here? Trends Ecol. Evol. 24, 201-207

10 Altman, I. and Byers, J.E. (2014) Large-scale spatial variation in parasite communities influenced by anthropogenic factors. Ecology 95, 1876-1887

11 Steeb, B. et al. (2013) Parallel exploitation of diverse host nutrients enhances Salmonella virulence. PLoS Pathog. 9, e1003301

12 Luijckx, P. et al. (2013) A matching-allele model explains host resistance to parasites. Curr. Biol. 23, 1085-1088

13 Ebert, E. et al. (2004) The evolution of virulence when parasites cause host castration and gigantism. Am. Nat. 164, S19-S32

14 Seppälä, O. et al. (2013) Are sick individuals weak competitors? Competitive ability of snails parasitized by a gigantism-inducing trematode. PLoS ONE 8, e79366

15 Abu Kwaik, Y. and Bumann, D. (2013) Microbial quest for food in vivo: 'nutritional virulence' as an emerging paradigm. Cell. Microbiol. 15, 882-890

16 Saliba, K.J. et al. (2006) Sodium-dependent uptake of inorganic phosphate by the intracellular malaria parasite. Nature 443, 582-585

17 Cressler, C.E. et al. (2014) Disentangling the interaction among host resources, the immune system and pathogens. Ecol. Lett. 17, 284-293

18 Coop, R.L. and Kyriazakis, I. (2001) Influence of host nutrition on the development and consequences of nematode parasitism in ruminants. Trends Parasitol. 17, 325-330

19 Cotter, S.C. et al. (2011) Macronutrient balance mediates trade-offs between immune function and life history traits. Funct. Ecol. 25, 186-198

20 Cornet, S. et al. (2014) Impact of host nutritional status on infection dynamics and parasite virulence in a bird-malaria system. J. Anim. Ecol. 83, 256-265

21 Brunner, F.S. et al. (2014) Protein-poor diet reduces host-specific immune gene expression in Bombus terrestris. Proc. R. Soc. B 281, 20140128

22 Povey, S. et al. (2014) Dynamics of macronutrient self-medication and illness-induced anorexia in virally infected insects. J. Anim. Ecol. 83, $245-255$
23 Howick, V. and Lazzaro, B. (2014) Genotype and diet shape resistance and tolerance across distinct phases of bacterial infection. BMC Evol. Biol. 14, 56

24 Mitchell, C.E. et al. (2003) Effects of elevated $\mathrm{CO}_{2}$, nitrogen deposition, and decreased species diversity on foliar fungal plant disease. Global Change Biol. 9, 438-451

25 Bruno, J.F. et al. (2003) Nutrient enrichment can increase the severity of coral diseases. Ecol. Lett. 6, 1056-1061

26 Voss, J.D. and Richardson, L.L. (2006) Nutrient enrichment enhances black band disease progression in corals. Coral Reefs 25, 569-576

27 Johnson, P.T.J. et al. (2007) Aquatic eutrophication promotes pathogenic infection in amphibians. Proc. Natl. Acad. Sci. U.S.A. 104, 15781-15786

28 Frost, P.C. et al. (2008) Responses of a bacterial pathogen to phosphorus limitation of its aquatic invertebrate host. Ecology 89, 313-318

29 Dallas, T. and Drake, J.M. (2014) Nitrate enrichment alters a Daphnia-microparasite interaction through multiple pathways. Ecol. Evol. 4, 243-250

30 Vale, P.F. et al. (2011) Epidemiological, evolutionary, and coevolutionary Implications of context-dependent parasitism. Am. Nat. 177, 510-521

31 Dordas, C. (2009) Role of nutrients in controlling plant diseases in sustainable agriculture: a review. In Sustainable Agriculture (Lichtfouse, E. et al., eds), pp. 443-460, Springer Netherlands

32 Smith, V.H. (1993) Resource competition between host and pathogen. Bioscience 43, 21-30

33 Aalto, S.L. and Pulkkinen, K. (2013) Food stoichiometry affects the outcome of Daphnia-parasite interaction. Ecol. Evol. 3, 1266-1275

34 Ebert, D. and Herre, E.A. (1996) The evolution of parasitic diseases. Parasitol. Today 12, 96-101

35 Pulkkinen, K. et al. (2014) Phosphorus limitation enhances parasite impact: feedback effects at the population level. BMC Ecol. 14, 29

36 Ben-Ami, F. et al. (2008) The effects of multiple infections on the expression and evolution of virulence in a Daphnia-endoparasite system. Evolution 62, 1700-1711

37 Garbutt, J. et al. (2011) Antagonistic competition moderates virulence in Bacillus thuringiensis. Ecol. Lett. 14, 765-772

38 Eswarappa, S.M. et al. (2012) Within-host dynamics of multi-species infections: facilitation, competition and virulence. PLoS ONE 7, e38730

39 Lacroix, C. et al. (2014) Environmental nutrient supply alters prevalence and weakens competitive interactions among coinfecting viruses. New Phytol. 204, 424-433

40 Smith, V.H. (2014) Nutrient supply differentially alters the dynamics of co-infecting phytoviruses. New Phytol. 204, 265-267

41 Rigaud, T. et al. (2010) Parasite and host assemblages: embracing the reality will improve our knowledge of parasite transmission and virulence. Proc. R. Soc. B 277, 3693-3702

42 Lange, B. et al. (2014) Diet quality determines interspecific parasite interactions in host populations. Ecol. Evol. 4, 3093-3102

43 McKenzie, V.J. and Townsend, A.R. (2007) Parasitic and infectious disease responses to changing global nutrient cycles. EcoHealth 4, 384-396

44 Colwell, R.R. (1996) Global climate and infectious disease: the cholera paradigm. Science 274, 2025-2031

45 Civitello, D.J. et al. (2015) Resources, key traits and the size of fungal epidemics in Daphnia populations. J. Anim. Ecol. Published online March 21, 2015. http://dx.doi.org/10.1111/1365-2656.12363

46 Vale, P.F. et al. (2013) Host nutrition alters the variance in parasite transmission potential. Biol. Lett. 9, 20121145

47 Ebert, D. et al. (2000) Dose effects and density-dependent regulation of two microparasites of Daphnia magna. Oecologia 122, 200-209

48 Gompper, M.E. and Wright, A.N. (2005) Altered prevalence of raccoon roundworm (Baylisascaris procyonis) owing to manipulated contact rates of hosts. J. Zool. 266, 215-219

49 Hall, S.R. et al. (2010) Variation in resource acquisition and use among host clones creates key epidemiological trade-offs. Am. Nat. $176,557-565$

50 Penczykowski, R.M. et al. (2014) Poor resource quality lowers transmission potential by changing foraging behaviour. Funct. Ecol. 28, 1245-1255 
51 Cory, J.S. and Hoover, K. (2006) Plant-mediated effects in insectpathogen interactions. Trends Ecol. Evol. 21, 278-286

52 de Roode, J.C. et al. (2011) Aphids indirectly increase virulence and transmission potential of a monarch butterfly parasite by reducing defensive chemistry of a shared food plant. Ecol. Lett. 14, 453-461

53 Seppälä, O. and Leicht, K. (2015) Quality attracts parasites: host condition-dependent chemo-orientation of trematode larvae. Funct. Ecol. Published online January 10, 2015. http://dx.doi.org/10.1111/ 1365-2435.12392

54 Parker, B.J. et al. (2011) Non-immunological defense in an evolutionary framework. Trends Ecol. Evol. 26, 242-248

55 Decaestecker, E. et al. (2014) Parasite and nutrient enrichment effects on Daphnia interspecific competition. Ecology Published online November 18, 2014. http://dx.doi.org/10.1890/14-1167.1

56 Wolinska, J. and King, K.C. (2009) Environment can alter selection in host-parasite interactions. Trends Parasitol. 25, 236-244

57 Sadd, B.M. (2011) Food-environment mediates the outcome of specific interactions between a bumblebee and its trypanosome parasite. Evolution 65, 2995-3001

58 Forde, S.E. et al. (2004) Adaptation varies through space and time in a coevolving host-parasitoid interaction. Nature 431, 841-844

59 Duffy, M.A. et al. (2012) Ecological context influences epidemic size and parasite-driven evolution. Science 335, 1636-1638

60 Jousimo, J. et al. (2014) Ecological and evolutionary effects of fragmentation on infectious disease dynamics. Science 344, $1289-1293$

61 Lopez-Pascua, L.D.C. and Buckling, A. (2008) Increasing productivity accelerates host-parasite coevolution. J. Evol. Biol. 21, 853-860

62 Kubinak, J.L. and Potts, W.K. (2013) Host resistance influences patterns of experimental viral adaptation and virulence evolution. Virulence 4, 410-418

63 Verreydt, D. et al. (2012) Dispersal-mediated trophic interactions can generate apparent patterns of dispersal limitation in aquatic metacommunities. Ecol. Lett. 15, 218-226

64 Boots, M. (2011) The evolution of resistance to a parasite is determined by resources. Am. Nat. 178, 214-220

65 Decaestecker, E. et al. (2013) Damped long-term host-parasite Red Queen coevolutionary dynamics: a reflection of dilution effects? Ecol. Lett. 16, 1455-1462

66 Råberg, L. et al. (2009) Decomposing health: tolerance and resistance to parasites in animals. Philos. Trans. R. Soc. Lond. B: Biol. Sci. 364 , $37-49$

67 Råberg, L. (2014) How to live with the enemy: understanding tolerance to parasites. PLoS Biol. 12, e1001989

68 Vale, P.F. et al. (2014) Limiting damage during infection: lessons from infection tolerance for novel therapeutics. PLoS Biol. 12, e1001769

69 Weider, L.J. et al. (2008) Stoichiometric differences in food quality: impacts on genetic diversity and the coexistence of aquatic herbivores in a Daphnia hybrid complex. Oecologia 158, 47-55

70 Jeyasingh, P.D. et al. (2009) Genetically-based trade-offs in response to stoichiometric food quality influence competition in a keystone aquatic herbivore. Ecol. Lett. 12, 1229-1237

71 Hall, S.R. et al. (2012) A power-efficiency trade-off in resource use alters epidemiological relationships. Ecology 93, 645-656

72 Frisch, D. et al. (2014) A millennial-scale chronicle of evolutionary responses to cultural eutrophication in Daphnia. Ecol. Lett. 17, 360-368

73 Rejmankova, E. et al. (2006) Freshwater community interactions and malaria. In Disease Ecology: Community Structure and Pathogen Dynamics (Collinge, S. and Ray, C., eds), pp. 90-104, Oxford university press

74 Turner, W.C. et al. (2014) Fatal attraction: vegetation responses to nutrient inputs attract herbivores to infectious anthrax carcass sites. Proc. R. Soc. B. 281, 20141785

75 Mittelbach, G.G. et al. (2001) What is the observed relationship between species richness and productivity? Ecology 82, 2381-2396

76 Keesing, F. et al. (2006) Effects of species diversity on disease risk. Ecol. Lett. 9, 485-498

77 Keesing, F. et al. (2010) Impacts of biodiversity on the emergence and transmission of infectious diseases. Nature 468, 647-652

78 Hall, S.R. et al. (2009) Friendly competition: evidence for a dilution effect among competitors in a planktonic host-parasite system. Ecology 90, 791-801
79 Johnson, P.T.J. and Thieltges, D.W. (2010) Diversity, decoys and the dilution effect: how ecological communities affect disease risk. J. Exp. Biol. 213, 961-970

80 Johnson, P.T.J. et al. (2013) Biodiversity decreases disease through predictable changes in host community competence. Nature 494, 230-233

81 Wood, C.L. and Lafferty, K.D. (2013) Biodiversity and disease: a synthesis of ecological perspectives on Lyme disease transmission. Trends Ecol. Evol. 28, 239-247

82 Wood, C.L. et al. (2014) Does biodiversity protect humans against infectious disease? Ecology 95, 817-832

83 Isbell, F. et al. (2013) Nutrient enrichment, biodiversity loss, and consequent declines in ecosystem productivity. Proc. Natl. Acad. Sci. U.S.A. 110, 11911-11916

84 Brown, S.P. et al. (2012) Evolution of virulence in opportunistic pathogens: generalism, plasticity, and control. Trends Microbiol. 20, 336-342

85 Wedekind, C. et al. (2010) Elevated resource availability sufficient to turn opportunistic into virulent fish pathogens. Ecology 91, $1251-1256$

86 Penczykowski, R.M. et al. (2014) Habitat structure and ecological drivers of disease. Limnol. Oceanogr. 59, 340-348

87 Hatcher, M.J. et al. (2006) How parasites affect interactions between competitors and predators. Ecol. Lett. 9, 1253-1271

88 May, R.M. and Anderson, R.M. (1978) Regulation and stability of host-parasite population interactions. II. Destabilizing processes. J. Anim. Ecol. 47, 249-267

89 Gerla, D.J. et al. (2013) Alternative states and population crashes in a resource-susceptible-infected model for planktonic parasites and hosts. Freshwat. Biol. 58, 538-551

90 Hall, S.R. et al. (2004) Stoichiometry and planktonic grazer composition over gradients of light, nutrients, and predation risk. Ecology 85, 2291-2301

91 Forshay, K.J. et al. (2008) Festering food: chytridiomycete pathogen reduces quality of Daphnia host as a food resource. Ecology 89, 26922699

92 Frost, P.C. et al. (2008) Bacterial infection changes the elemental composition of Daphnia magna. J. Anim. Ecol. 77, 1265-1272

93 Vázquez-de-Aldana, B.R. et al. (2013) Fungal endophyte (Epichloë festucae) alters the nutrient content of Festuca rubra regardless of water availability. PLoS ONE 8, e84539

94 Ponton, F. et al. (2011) Nutritional immunology: a multi-dimensional approach. PLoS Pathog. 7, e1002223

95 Graham, R.I. et al. (2014) Locusts increase carbohydrate consumption to protect against a fungal biopesticide. J. Insect Physiol. 69, 27-34

96 Bernot, R.J. (2013) Parasite-host elemental content and the effects of a parasite on host-consumer-driven nutrient recycling. Freshw. Sci. 32, 299-308

97 Jeyasingh, P.D. et al. (2014) Testing the ecological consequences of evolutionary change using elements. Ecol. Evol. 4, 528-538

98 Roy Chowdhury, P. et al. (2014) Functional genomics of intraspecific variation in carbon and phosphorus kinetics in Daphnia. J. Exp. Zool. 321A, 387-398

99 Jeyasingh, P.D. et al. (2011) How do consumers deal with stoichiometric constraints? Lessons from functional genomics using Daphnia pulex. Mol. Ecol. 20, 2341-2352

100 Sterner, R.W. and Elser, J.J. (2002) Ecological Stoichiometry: The Biology of Elements from Molecules to the Biosphere, Princeton University Press

101 Elser, J.J. et al. (2000) Nutritional constraints in terrestrial and freshwater food webs. Nature 408, 578-580

102 Hessen, D.O. et al. (2013) Ecological stoichiometry: an elementary approach using basic principles. Limnol. Oceanogr. 58, 2219-2235

103 Lukas, M. et al. (2011) Growth rate hypothesis does not apply across colimiting conditions: cholesterol limitation affects phosphorus homoeostasis of an aquatic herbivore. Funct. Ecol. $25,1206-1214$

104 Malzahn, A.M. et al. (2010) Differential effects of nutrient-limited primary production on primary, secondary or tertiary consumers. Oecologia 162, 35-48

105 Civitello, D.J. et al. (2012) Potassium stimulates fungal epidemics in Daphnia by increasing host and parasite reproduction. Ecology 94 380-388 